\title{
Optimisation BASEd on Simulation: A PATIENT Admission SCHEDUling Problem in a RADiology DEPARTMENT CASE STUDY
}

\author{
${ }^{1}$ V.K.Manupati, ${ }^{2}$ G.Mourya Teja, ${ }^{2}$ Zakir Hussain S K, ${ }^{2}$ Y.Sandeep and \\ ${ }^{2}$ A.Akhil Varma \\ ${ }^{1}$ Associate Professor, Department of Mechanical engineering, KL University, \\ Vijayawada, AP
}

\begin{abstract}
In this paper we have considered a Patient Admission scheduling problem for reducing the patients waiting time by improving the resources effectively. Recently, appointment scheduling systems in health care services are at most important to respond to an ever increasing demand for care its utilization is focused on enhancing the usage of human resources, medical equipment and reducing the patient waiting time. We have carried out a case on patient admission scheduling problem in radiology department. A mathematical model has been developed as a linear programming model to optimally solve this problem. The proposed model is hard to solve with traditional methods due to its computational complexity, to achieve the optimal solutions in a reasonable time an evolutionary algorithm based multi-objective Non dominated sorting genetic algorithm is adopted. With the data collected from the daily observations the experimentation has been conducted the obtained results showed average reductions of $7 \%$ of the total completion and $34 \%$ on the patients total waiting time
\end{abstract}

\section{KEYWORDS}

Patient admission scheduling, appointment scheduling systems, Non dominated sorting genetic algorithm, patient waiting time.

\section{INTRODUCTION}

Recently, health care services is one of the pressing area that needs to be highlighted for the betterment of society. In order to increase the awareness for preventative care and to serve the patients in an effective manner the reorganization of present health care systems is essential. Recent increase in demand for health care services and lack of ability to handle the no of health care providers leads to inefficiency of the system [1]. To handle the above mentioned problem it is necessary to implement the effective and efficient methodologies. Increasing waiting list and the ageing of the population are the two most important problems now a days the health care organizations are looking for. In this context, we have considered a radiology department to carry the patient admission scheduling problem (PAS).

The Admission Scheduling (AS) problem is first introduced by Bailey [2]. The solution for the problem concluded that the schedule of patients in regular intervals can produce better results and it is equal to the average consultation time. The AS problem in a healthcare environment was first addressed by cayirli and veral [3]. Here, authors have solved the appointment schedule in a healthcare environment with an objective function having multiple performance measures is 
International Journal on Cybernetics \& Informatics (IJCI) Vol. 4, No. 2, April 2015

optimized and highlight several issues for both static and dynamic cases. In this work, a PAS problem is considered to improve the comfort of the patience.

In general, The PAS problem in a radiology department is considered in order to minimize patience, length of stay in waiting list while reducing the cost and increasing the quality of care. Primarily, we considered a case of radiology department for solving the patient admission scheduling problem. To carry the problem a clinic has been identified and then observed various departments and its operations involved in the clinic later for a period of four months data was collected and statistical analysis was conducted. Moreover a mathematical model has been developed in subjected to constraints by identifying the objectives as total completion time and total waiting time of the patient. Due to the problems complexity and its influence on recent researchers, we are interested to carry this problem to find the efficient and feasible solutions, particularly in the context of PAS environment. Therefore, it is necessary to introduce efficient and effective methods to schedule the mentioned problem. As the proposed problem is multiobjective in nature we used a multi-objective evolutionary algorithm to solve the above mentioned problem.

The remainder of this paper is organized as follows. The problem description with mathematical model subjected to constraints is developed and its notations are detailed in Section 2. Section 3 the framework of multi-objective evolutionary algorithm is presented. In Section 4 the mapping of problem with proposed multi-objective based NSGA-II algorithm is detailed and solved. The experimentation with collected data is presented and their results are presented in section 5 . The paper concludes with section 6 which suggests the directions of future work.

\section{Mathematical Representation And Problem Description}

In this paper, the addressed problem is map as a queuing system with the objective of improving the performance measures such as minimizing patients' waiting time. The queuing system is divided into number of stages and number of servers. The number of stages and servers are defined as: 1. Number of stages and 2. Multistage system. Chel et al. [4] considered four stages in the analysis of surgery admission. Conelly and Bair [5] explored the potential of discrete event simulation in the operation analysing an emergency department. In their work multistage system was considered as each patient was modelled as a set of instructions that defines a series of individual activities that must be completed in a given order before patient leaves the emergency department. The number of stages are defined in this work as, a. Single stage system and b. Multistage system. Where, in single stage system the patients maintain in a single queue. The majority of the studies in a literature represent single-stage problems. Schemes, illustrating single-stage systems, are provided in Figure 1(a) and (b)

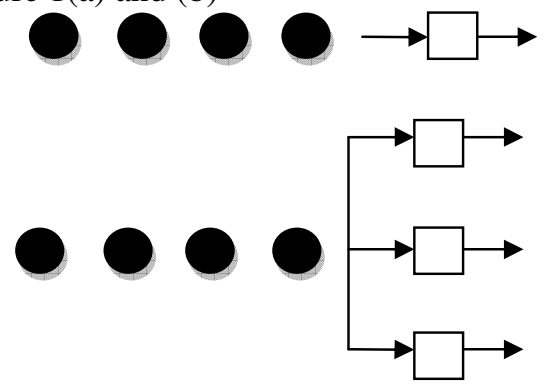

Figure1. (a) Single stage/Single server system (b) Single-stage/Multi server system 
International Journal on Cybernetics \& Informatics (IJCI) Vol. 4, No. 2, April 2015

Whereas in a multistage system, parents queue for a multiple stages such as registration, examination and check out schemes that are shown in Figure.2 (a) and (b). A multistage system was considered by Garg et al. [6]. In this work, a hospital scenario was studied and patient pathway was modelled to consider different phases of care, such as acute, treatment and rehabilitation. In the flow modelled by the Granja et al. [7], three stages were considered in the patient pathway in radiology admission, examination and billing.

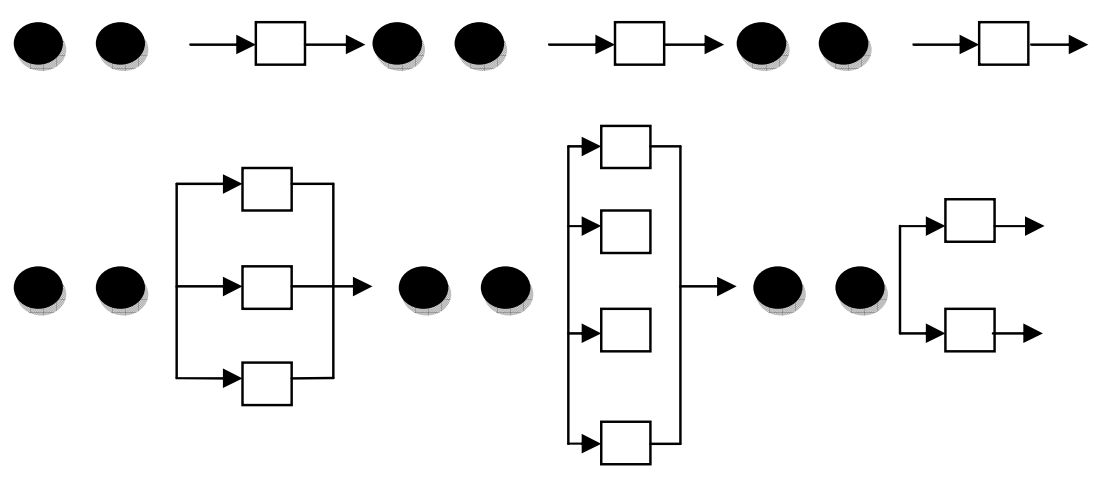

Figure2. (a) Patient path way as a Single stage schedule system (b) Patient path way represented with Multi stage schedule system

The patience path way is mapped with queuing system is depicted in Fig. 2. It shows different types of stages and their functions with respect to patient admission scheduling problem. The functionalities of these systems and its representation is mentioned in the literature [8, 9]. In multi-server system more than one server with same capabilities is considered in appointment system [10-12] the scheduling algorithm decides to which server each patient is queuing. Schemes illustrating multi-server systems are provided in Fig.2 (a) and Fig.2 (b) a multi stage schedule system was considered by a Hoot et al. [13].

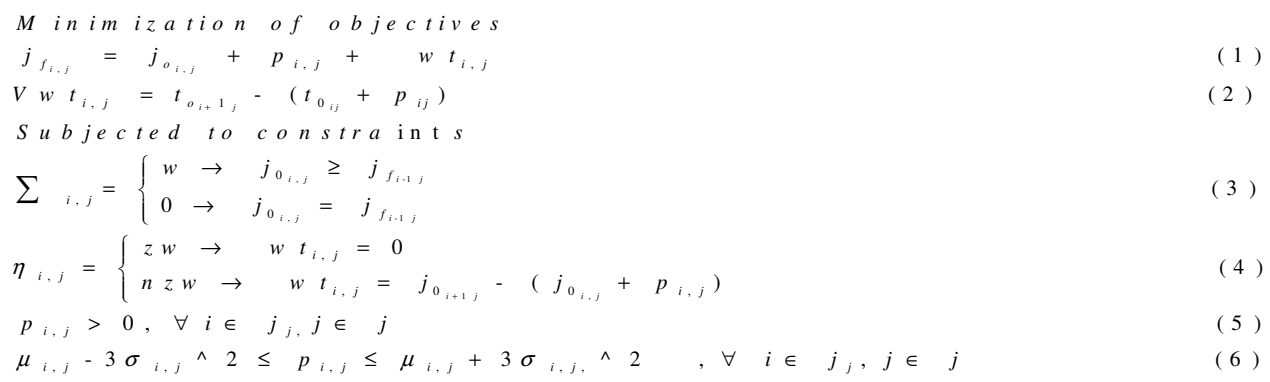

\section{CASE Study}

This article approaches an AS problem in a Private hospital. The clinic is integrated within the hospital and there are more number of service requests with scheduled patients delivered by the clinic. In the work carried out in the beginning of the project, a non-significant amount of patient delays are observed. This may be due to social environment of the clinic. As the hospital is situated in a rural area and serves a vast area, due to social factors the patients tend to arrive early for the appointment, often several hours before the scheduled time. Thus it was not possible to characterize this environmental factor. 
International Journal on Cybernetics \& Informatics (IJCI) Vol. 4, No. 2, April 2015

The considered clinic in this paper provides various services on Computed Tomography (CT), MRI, Orthopantomography (OT), Densitometry (PX), Mammography (MG) and Ultrasound (US). It has 10 daily working hours and is composed of human, physical and technical resources, such as: Physicians; Technicians; Assistants; Administration; Waiting room; changing room; Control rooms; Examination rooms; Modalities. There are total four dressing rooms can be shared by both modalities. Both CT and MRI modalities are taken care by same human and physical resources. Whereas, the call for examinations is made by the same assistant, who directs to the dressing rooms. PX/MG and OT/US share one technician that executes the examination. The framework of detail case and its procedure is shown in Figure 3.

In this case, patients requests for appointments are by two modes i.e., either by phone or in person. At the moment of request, the reception staff can assist to schedule patient appointment for a day and time according to availability of the schedule time. The size of the time block, i.e., processing time depends upon the kind of modality the patient is referred for admission. During the day of the appointment from the above mentioned two modes the patient refers to the clinic for admission. However, patient should wait for some time after arrival at waiting hall until the respective modality assistant called for examination. The service is provided to the patient when the necessary resources are available and according to their turn and their rescheduled time made by the technicians and based on their empirical knowledge on the type of examination.

The processes implemented at the studied clinic were modelled by the loco observation and characterized according to the parameters described in following sections. The parameters defined based on optimum workflow, to ensure the process models accuracy. These parameters allow describing of the real case, while maintaining the modelling flexibility and robustness.

\section{Multi-ObJective Nsga-Ii Algorithm}

Recently, multi-objective optimization in health care problems captured the interest of many researchers. The feasibility of the proposed approach is verified with a multi-objective based GA (NSGA-II) algorithm [9] to find the feasible solutions for proposed problem. The operators play a crucial role in the algorithm for generating of the better quality solutions. In order to find the better quality solutions, it is necessary to fine tune the parameters such as crossover and mutation in better manner. Therefore, the authors have said the ranges of operators i.e. crossover as 0.65 to 0.95 and mutation as 0.1 to 0.01 . By maintaining these ranges with the termination criteria as 100 iterations we have run the algorithm on an average of 10 per run. In this manner we improved the system performance better than the existing one.

In this research, we considered a multi-objective NSGA-II to generate optimal solutions. Goldberg was the first to suggest the non-dominated sorting genetic algorithm (NSGA) and Srinivas and Deb were the first to execute it. As NSGA differs from well-known simple genetic algorithm is only in the way the selection operator works. Some drawbacks that need to be highlighted for NSGA are: (a) huge time for obtaining the quality of solutions, (b) not obtaining the elitist operation (c) lack of tenability for Pareto fronts. With the advent of NSGA-II, the above mentioned drawbacks have been resolved. We first apply a classical non-dominated sorting genetic algorithm-II (NSGA-II) [14] and its schematic procedure is depicted in Figure 4 which includes Pareto ranking and crowding distance mechanism for selection of the individuals. 
International Journal on Cybernetics \& Informatics (IJCI) Vol. 4, No. 2, April 2015

Figure 3. Flow chart of the proposed procedure of health systems

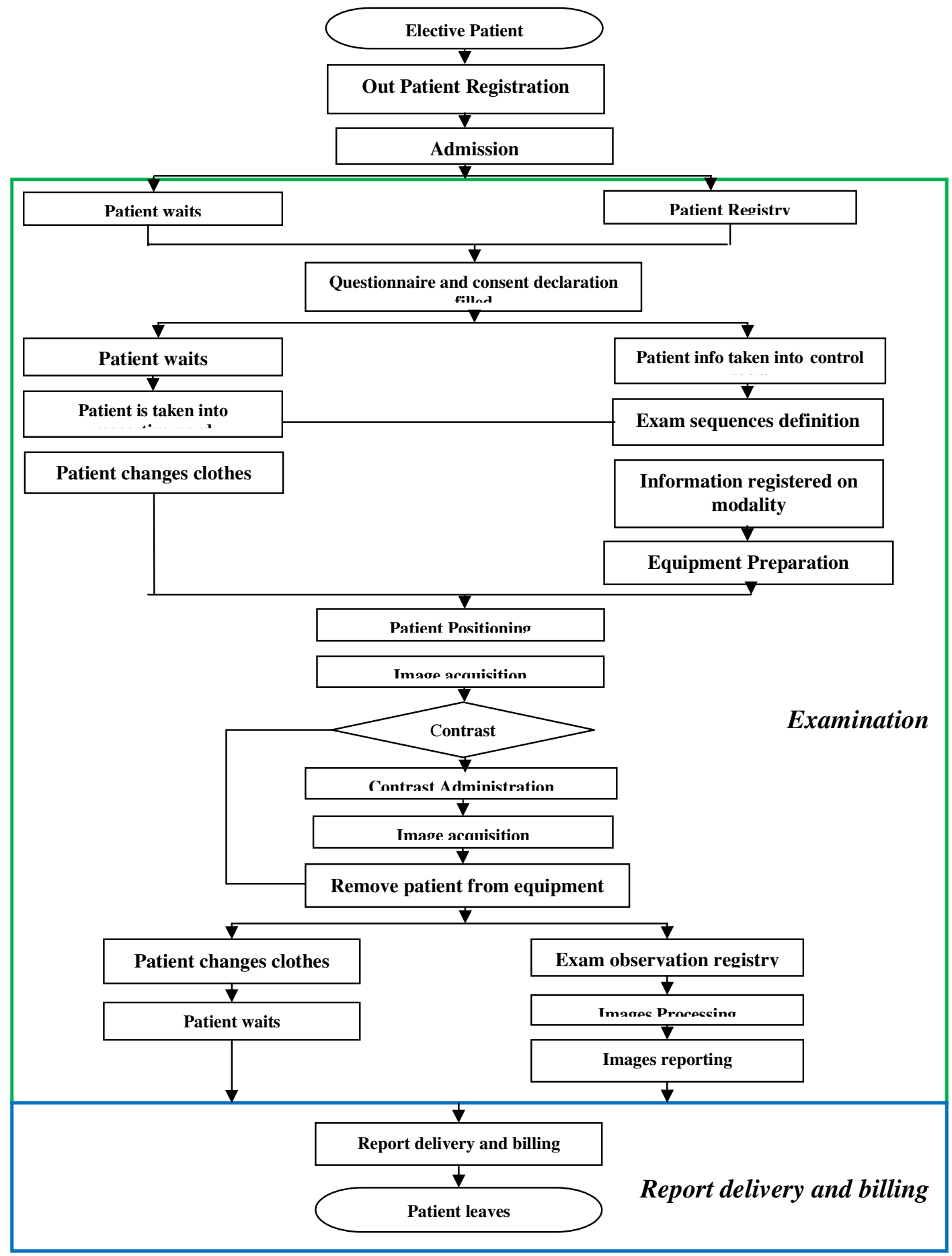


International Journal on Cybernetics \& Informatics (IJCI) Vol. 4, No. 2, April 2015

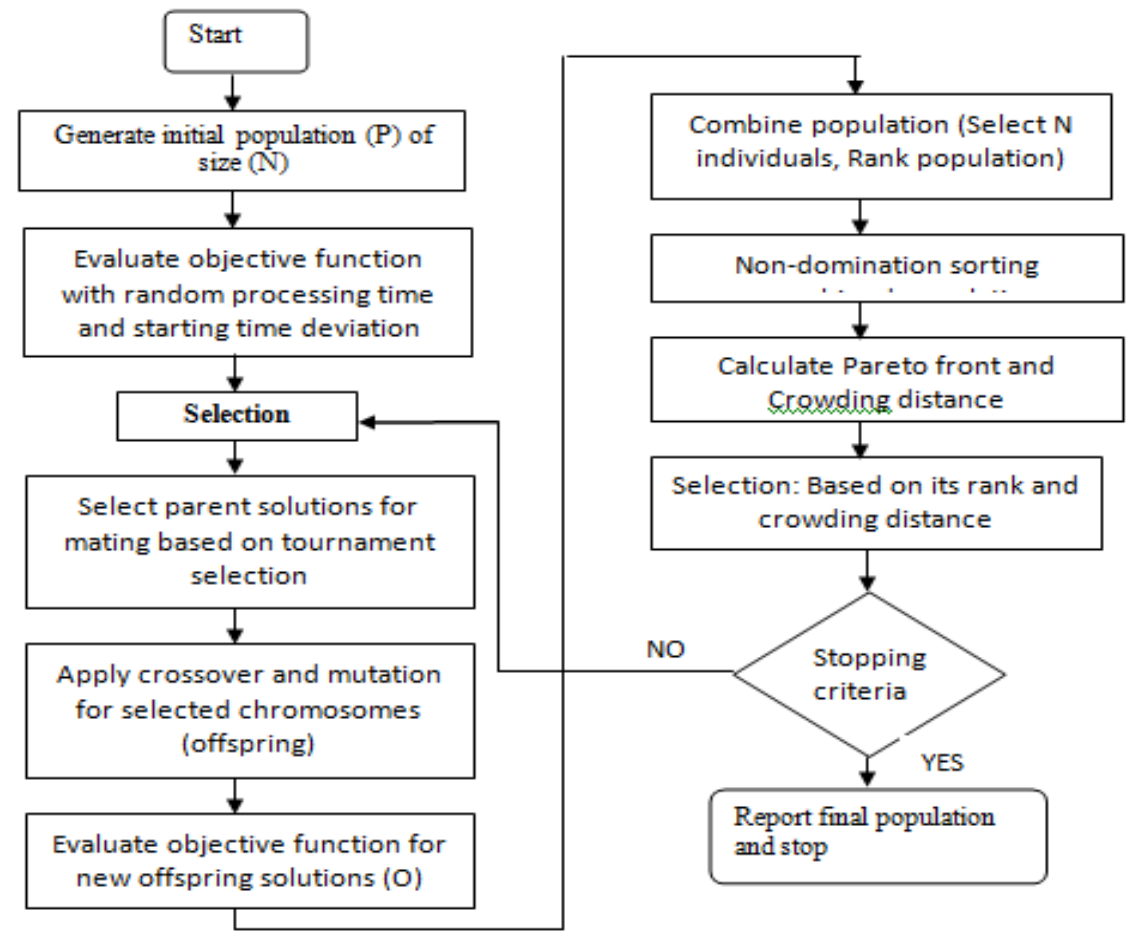

Figure 4. Schematic procedure for processing NSGA-II

\section{EXPERIMENTATION}

To understand the behaviour of the resources and their utilization at the studied clinic, 39 exam types were identified and then investigated for a period of 4 months, data was collected by the observation of the daily work. The work load data is represented in Figure 5.

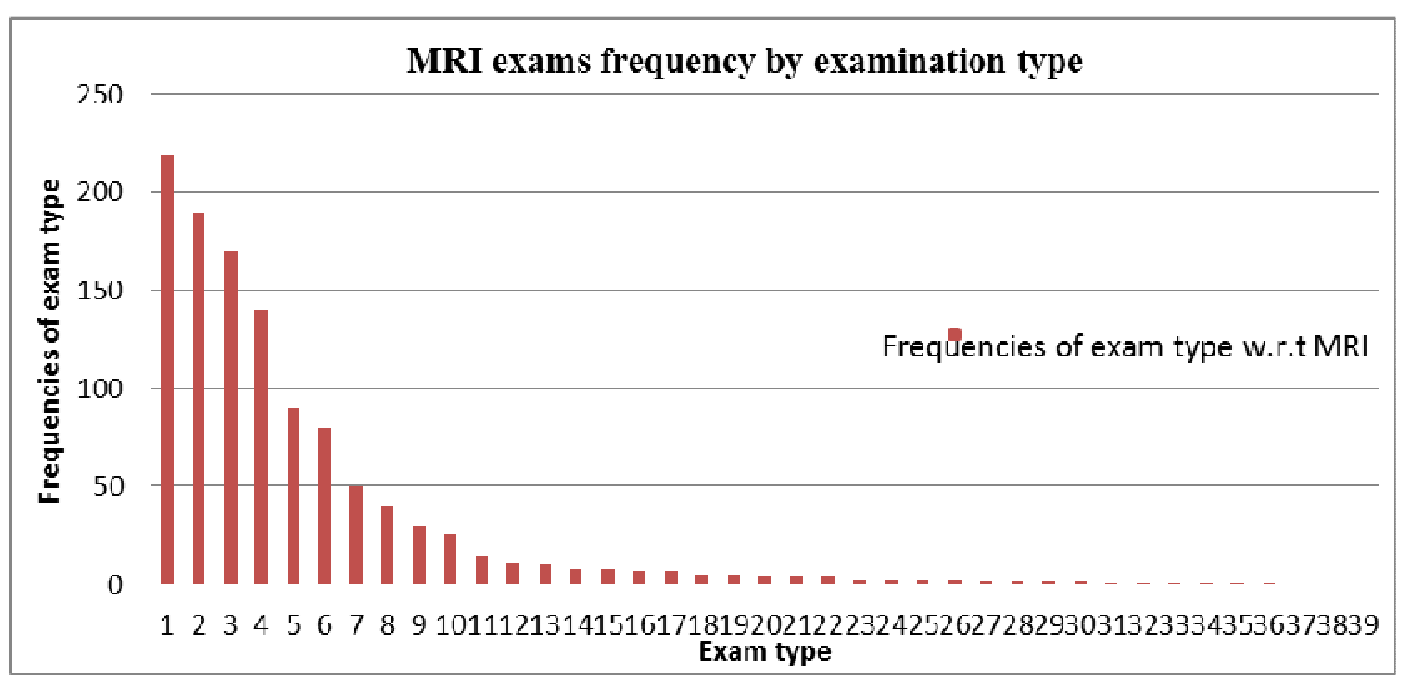

Figure 5. MRI exams types and their frequencies 
International Journal on Cybernetics \& Informatics (IJCI) Vol. 4, No. 2, April 2015

Over six exam types depicts that $80 \%$ of the total work load (cervical, lumbar, knee, brain, breast and shoulder). This lead to limitation of the exams observed, during the second phase of the six exam types mentioned above, given the amount of perception time required to obtain a sample that would be statistically illustrated.

Table 1. Statistical Analysis of the work flow of Main Examinations in the Clinic

\begin{tabular}{|l|r|r|r|}
\hline Parameter & Task & & \\
\hline & 1st cloth change(min) & Prepare Equipment (min) & $\begin{array}{l}\text { Patient } \\
\text { positioning(min) }\end{array}$ \\
\hline & & & 0.64 \\
\hline Average & 2.32 & 0.55 & 1.98 \\
\hline Median & 1.95 & 0.31 & 0.65 \\
\hline Standard deviation & 0.9 & 1.36 & 1.97 \\
\hline & Contrast (min) & Remove patient (min) & 1.85 \\
\hline Average & 2.09 & 1.19 & 0.9 \\
\hline Median & 1.94 & 0.67 & \\
\hline Standard deviation & 0.74 & & \\
\hline
\end{tabular}

Table 2. Statistical Analysis of the Image Acquisition Task by Examination Type

\begin{tabular}{|c|c|c|c|c|}
\hline Parameter & Exam with contrast & & & \\
\hline & Cervical(min) & Lumbar (min) & Brain (min) & Breast(min) \\
\hline Average & 20.42 & 26.51 & 26.72 & 25.92 \\
\hline Median & 19.52 & 25.52 & 25.17 & 26.65 \\
\hline Standard deviation & 4.48 & 6.06 & 8.25 & 4.91 \\
\hline Average & 18.02 & 15.71 & 15.77 & 16.25 \\
\hline Median & 18.3 & 15.61 & 15.57 & 15.81 \\
\hline Standard Deviation & 3.8 & 1.27 & 1.34 & 2.8 \\
\hline
\end{tabular}

The statistical analysis on the clinic work flow of main examinations is given in Table 1. In Table 1 , starting from patient dress change to patient task completion and its average, median, and standard deviations are shown. Similarly, in Table 2 the image acquisition task of different examination types are shown in Table 2. Moreover, after finding of statistical results the simulation experiments are conducted with NSGA-II algorithm. In the Figure.6 the Pareto optimal curve for the multi objective functions such as total completion time and total waiting time of the patient has been shown, also the optimal results is shown in Table 3. The mentioned algorithms are coded with MATLAB software and the problem is tested on Intel ${ }^{\circledR}$ Core ${ }^{\mathrm{TM}} 2$ Duo CPU T7250 @2.00GHz, 1.99 GB of RAM.

Table 3. Simulation Results of Nsga II

\begin{tabular}{|l|l|}
\hline Performance measures & NSGA II \\
\hline Total competition time & 86 \\
Total waiting & 9 \\
\hline $\mathrm{F}_{\mathrm{obj}}$ & 95 \\
\hline
\end{tabular}


International Journal on Cybernetics \& Informatics (IJCI) Vol. 4, No. 2, April 2015

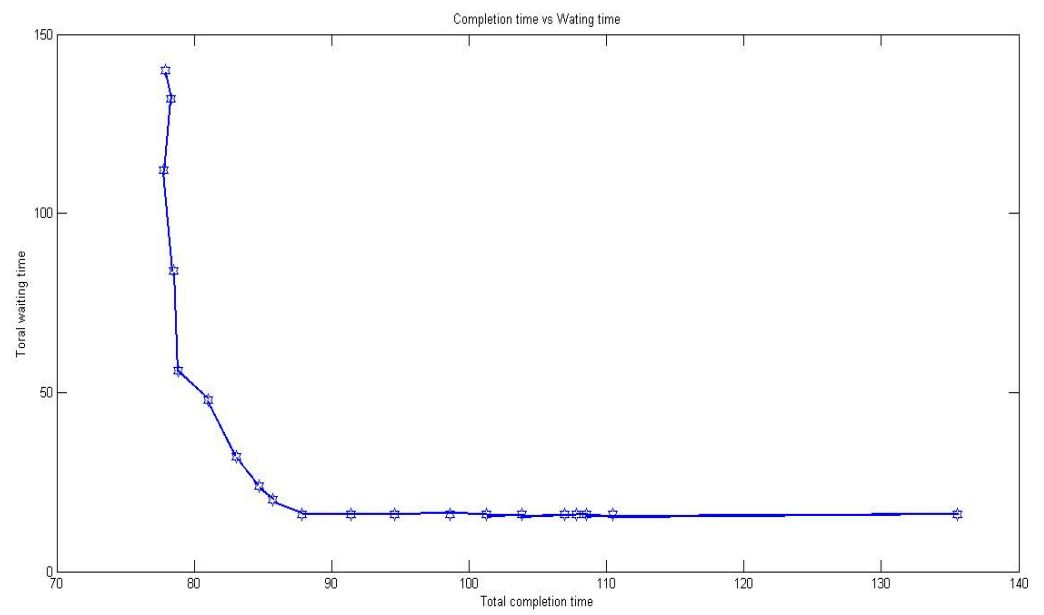

Figure 6. Pareto optimal graph of make span and waiting time with NSGA-II algorithm

\section{CONCLuSiON}

In this phase patient admission scheduling problem (PAS) was implemented in three stages: work flow definition, data collection and simulation and optimization via NSGA-II. First, it was achieved through a comprehensive overview of the clinic work flows and to identify possible tasks that weren't taken in to picture in the previous definition of an ideal workflow. The data at this stage created the principles for the elaboration of a detailed checklist, universal to all providers that systematizes the significant data collection. In the following, the used methodology is endeavoured for the modality .A drawback of simulation tools, based on linear programming, is that this approach attempts to solve the PAS problem as a queuing system, by generating an empirical fit to the framework defined in the process model. Such limitations were minimized by the modelled methodology, the system modules that defines the framework. The radiology department was studied in terms of efficiency and production quality, denoted by total completion time and total patient waiting time, respectively. The patient cycle i.e. simulated for optimum solutions and the patient call order imposed by the technicians are similar. If the type of examination is considered during the patient admission, and as a result patients with similar exams are admitted continuously. Then the patient waiting time will be minimized, when the technician refers the patient sequence on the day of examination to enhance the internal sequence of examination these results on an extension of patient waiting time, reckoning that male or female. Therefore it is presumed that the type of examination must be contemplated in patient admission.

\section{REFERENCES}

[1] Fei, H., Meskens, N., Chu, C. (2010). A planning and scheduling problem for an operating theatre using an open scheduling strategy. Computers and Industrial Engineering, 58, 221-230.

[2] Bailey NT. A study of queues and appointment systems in hospital out-patient departments, with special reference to waiting-times. J Roy Stat Soc Ser B Met 1952; 14(2):185-99.

[3] Cayirli T, Veral E. Outpatient scheduling in health care: a review of literature. Prod Oper Manage 2003; 12(4):519-49. 
International Journal on Cybernetics \& Informatics (IJCI) Vol. 4, No. 2, April 2015

[4] Chen N, Zhan Z, Zhang J, Liu O, Liu H. A genetic algorithm for the optimization of admission scheduling strategy in hospitals. In: 2010 IEEE congress on evolutionary computation (CEC), Barcelona; 2010.

[5] Connelly L, Bair A. Discrete event simulation of emergency department activity: a platform for system-level operations research. Acad Emerg Med 2004; 11(11):1177-85.

[6] Garg L, McClean S, Meenan B, Millard P. A non-homogeneous discrete time Markov model for admission scheduling and resource planning in a cost or capacity constrained healthcare system. Healthc Manage Sci 2010; 13(2):155-69.

[7] Granja C, Mendes J, Janela F, Soares J, Mendes A. Optimisation-based on simulation: a diagnostic imaging department case-study. In: Second international conference on information, process, and knowledge management (eKNOW), Saint-Marteen; 2010.

[8] Diwas SK, Terwiesch C. The effects of focus on performance: evidence from California Hospitals. Manage Sci 2011; 57(11):1897-912.

[9] J.M. Molina-Pariente, V. Femandez-Viagas, J.M. Framinan Integrated operation Room planning and scheduling problem with assistant surgeon dependent surgery duration.Compute and industrial engineering 2015.

[10] Liu N, Ziya S, Kulkarni V. Dynamic scheduling of outpatient appointments under patient no-shows and cancellations. Manuf Serv Oper Manage 2010; 12(2):347-64.

[11] Alaeddini A, Yang K, Reddy C, Yu S. A probabilistic model for predicting the probability of no-show in hospital appointments. Health Care Manage Sci 2011; 14(2):146-57.

[12] Ceschia, Sara, and Andrea Schaerf. "Local search and lower bounds for the patient admission scheduling problem." Computers \& Operations Research38.10 (2011): 1452-1463.

[13] Hoot NR, LeBlanc LJ, Jones I, Levin SR, Zhou C, Gadd CS, et al. Real-time evaluation. J Am Med Inform Assoc 2009; 16(3):338-45.

[14] Deb, Kalyanmoy, et al. "A fast elitist non-dominated sorting genetic algorithm for multi-objective optimization: NSGA-II." Lecture notes in computer science1917 (2000): 849-858. 